\section{PSICOLOGÍA IBEROAMERICANA}

\section{Psicología Iberoamericana}

ISSN: 1405-0943

psicología.iberoamericana@uia.mx

Universidad Iberoamericana, Ciudad de

México

México

Robles Ojeda, Francisco Javier; Galicia Moyeda, Iris Xóchitl; Sánchez Velasco, Alejandra;

Pichardo Hernández, Amalia

Afectividad y dimensiones temporales asociadas a la depresión en estudiantes de bachillerato

Psicología Iberoamericana, vol. 23, núm. 1, enero-junio, 2015, pp. 40-47

Universidad Iberoamericana, Ciudad de México

Distrito Federal, México

Disponible en: http://www.redalyc.org/articulo.oa?id=133944230005

Cómo citar el artículo

- Número completo

- Más información del artículo

- Página de la revista en redalyc.org

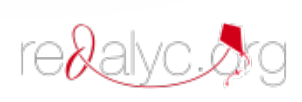

Sistema de Información Científica

Red de Revistas Científicas de América Latina, el Caribe, España y Portugal Proyecto académico sin fines de lucro, desarrollado bajo la iniciativa de acceso abierto 


\title{
Afectividad y dimensiones temporales asociadas a la depresión en estudiantes de bachillerato
}

\author{
Affection and temporary aspects linked to depression \\ in pre-university students
}

Francisco Javier Robles Ojeda

Iris Xóchitl Galicia Moyeda

Alejandra Sánchez Velasco

Amalia Pichardo Hernández ${ }^{1}$

\section{RESUMEN}

La depresión es un trastorno del estado de ánimo que impacta negativamente en la vida cotidiana de los adolescentes. El estado afectivo es un elemento que se ha encontrado asociado con la depresión. Asimismo, determinadas orientaciones temporales inciden en la sensación de bienestar o malestar emocional. La presente investigación indaga la correlación de estas variables en estudiantes de bachillerato, además de evaluar la capacidad predictora de la afectividad y orientación temporal con respecto a la depresión. Los resultados muestran una correlación entre la depresión con la afectividad estado y determinadas orientaciones personales, además de destacar como predictores de la depresión a la afectividad-estado y las dimensiones temporales de pasado negativo y presente hedonista.

Palabras clave: depresión, adolescencia, psicología positiva, afectividad, perspectiva temporal

\section{ABSTRACT}

Depression is an emotional disorder that has a negative impact on the daily life of adolescents. The emotional state is an element that has been linked to depression. Specific and temporary factors have an impact on the sensation of emotional wellbeing or distress. This paper explores the correlation between these variables among pre-university students, and also evaluates the predictive capacity of temporary issues and emotions in regard to depression. The results show a correlation between depression and emotional states, and specific personal issues, as well as highlighting how Affection-State and temporary issues of Negative Past and Hedonist Present are predictors of depression.

Keywords: depression, adolescence, positive psychology, affection, temporary perspective.

Fecha de recepción: 13 de mayo de 2014 -Fecha de aceptación: 16 de noviembre de 2014

${ }^{1}$ Francisco Javier Robles Ojeda. Facultad de Estudios Superiores Iztacala. e-mail: solucion20@hotmail.com Iris Xóchitl Galicia Moyeda. Facultad de Estudios Superiores Iztacala. e-mail: iris@unam.mx

Alejandra Sánchez Velasco. Facultad de Estudios Superiores Iztacala. e-mail: aavale@servidor.unam.mx

Amalia Pichardo Hernández. Colegio de Ciencias y Humanidades Azcapotzalco. e-mail: mayitaph@hotmail.com 


\section{INTRODUCCIÓN}

\section{Depresión}

La depresión es un trastorno mental que comprende un conjunto de signos y síntomas. De acuerdo con la quinta edición del Manual Diagnóstico de Enfermedades Mentales (DSM-5) se caracteriza por la presencia de un estado de ánimo deprimido y/o pérdida de placer en casi cualquier actividad por un lapso mínimo de dos semanas, produciéndose un malestar o deterioro significativo en la vida de la persona (Schimelpfening, 2013). Además de estos dos síntomas se deben presentar otros tres de la siguiente lista: pérdida importante de peso sin hacer dieta o aumento de peso, insomnio o hipersomnia, agitación o retraso psicomotor, fatiga o pérdida de energía, sentimientos de inutilidad o culpabilidad, disminución de la concentración, pensamientos de muerte recurrentes (Asociación Psiquiátrica Americana, 2014).

Por su parte, el modelo cognitivo propuesto por Beck sugiere que la depresión surge a partir de una secuencia de elementos: experiencias negativas tempranas, las cuales generan un conjunto de esquemas y actitudes disfuncionales que conforman la denominada tríada cognitiva de la depresión: a) visión negativa de sí mismo; b) visión negativa del entorno; y c) visión negativa del futuro o desesperanza (Rivadeneira, Dahab \& Minici, 2013). Al producirse esto y afrontar el sujeto acontecimientos críticos en su vida, se activarían de nuevo estos esquemas y actitudes, produciendo finalmente sesgos de procesamientos o pensamientos negativos (por ejemplo, ante un logro no alcanzado, pensar en que "soy un total fracaso y me merezco que la vida me castigue”), lo que a su vez genera la depresión (Vázquez, Hervás \& Hernangómez, 2008).

La depresión es considerada como uno de los principales trastornos mentales que se presentan a lo largo de la vida. Berenzon, Lara, Robles y Medina-Mora (2013) refieren que a nivel mundial afecta aproximadamente a 350 millones de personas y que los índices de prevalencia en algunos casos llegan hasta el $21.4 \%$. Entre los principales grupos afectados por la depresión se encuentran los adolescentes, quienes son una de las principales poblaciones de riesgo para la aparición y desarrollo de este trastorno. Según la Encuesta Nacional de Epidemiología Psiquiátrica (ENEP)
(Medina-Mora et al., 2003), se señala que $2 \%$ de la población en México ha sufrido un episodio de depresión mayor antes de los 18 años de edad y que este grupo presenta en promedio siete episodios depresivos a lo largo de su vida.

Las consecuencias de la depresión en la vida de las personas son múltiples, ya que dependiendo de la intensidad de los síntomas éstos afectarán de manera parcial o total su funcionalidad en las principales áreas de su vida, ya sea familiar, escolar o laboral. De acuerdo con Berenzon, et al., (2013), en México la depresión llega a tener efectos discapacitantes mayores e incluso algunas enfermedades como las cardiacas, respiratorias o la diabetes. Esto coincide con la proyección que hace la Organización Mundial de la Salud, que prevé que para el año 2020 la depresión sea la segunda causa de discapacidad en el mundo (Medina-Mora et al., 2003).

\section{Afectividad}

Un elemento íntimamente relacionado con la presencia de síntomas depresivos es la afectividad o estado afectivo. La afectividad es un concepto que engloba los siguientes tres elementos: a) estado de ánimo, conformado por estados o tonalidades afectivas que caracterizan a una persona y su relación con el mundo en un periodo determinado; b) emociones, caracterizadas por un conjunto de respuestas conductuales, afectivas, somáticas y cognitivas que se viven de manera intensa y se dan en un corto tiempo ante un estímulo específico; y c) evaluaciones afectivas, formadas por juicios agradable-desagradable que hace un sujeto acerca de algunos aspectos en su vida (Fernández, Ubillos \& Zubieta, 2000; Páez \& Carbonero, 1993). En general, se pueden identificar dos dimensiones principales de la afectividad, las cuales representan polos distintos y relativamente independientes de este concepto: afecto positivo y afecto negativo. La dimensión positiva se caracteriza por emociones placenteras y sensaciones de energía, entusiasmo, confianza, motivación incrementada, logro y éxito. En contraste, la dimensión negativa se manifiesta por emociones displacenteras, miedos, inhibición, inseguridad, frustración, fracaso y culpa. 
En cuanto a su relación con la depresión, algunas investigaciones señalan que una alta afectividad positiva es un factor protector ante la depresión, además de estar asociado con un buen rendimiento en la vida de las personas -ya sea laboral o escolar- y optimismo ante la vida. Mientras que una alta afectividad negativa está asociada con la presencia de síntomas de depresión, estrés y ansiedad (Fernández et al., 2000; Moral de la Rubia, 2011). En este mismo sentido, Clark y Watson han identificado que la depresión se podría distinguir por una combinación de altos índices de afectividad positivos y bajos de afectividad negativa -a diferencia de otros trastornos, como la ansiedad, donde sólo se presentan altos niveles de afectividad negativa (Torres, Carbonell, Pérez \& Riquelme, 2013). Estos hallazgos han sido encontrado en otras investigaciones realizadas con poblaciones de niños (Sandín, 2003) y ancianos (Torres et al., 2013).

\section{Orientación temporal}

La perspectiva u orientación temporal es un elemento que algunos investigadores han planteado como un elemento a desencadenar determinada tendencia emocional en las personas (Zimbardo \& Boyd, 2009). Este concepto se puede definir como la tendencia personal hacia un estado temporal determinado -pasado, presente o futuro- (Díaz-Morales, 2008). De acuerdo con Zimbardo y Boyd (2009), dicha tendencia está determinada en buena parte por factores culturales que influyen en el comportamiento individual y social. Estos autores postulan cinco dimensiones principales de la orientación personal: a) Pasado positivo: se recuerda con añoranza y alegría el pasado; b) Pasado negativo: predominan actitudes pesimistas del pasado; c) Presente hedonista o placentero: se satisfacen deseos en el aquí y el ahora, en ocasiones sin tomar en cuenta riesgos; d) Presente fatalista: visión pesimista del futuro asociada a incertidumbre del momento actual; y e) Futuro: planificación y realización de acciones para alcanzar metas en un futuro que se prevé esperanzador (Díaz-Morales, 2008; Oyanadel \& BuelaCasal, 2010; Zimbardo \& Boyd, 2009).

Investigaciones (Díaz-Morales, 2008; Oyanadel \& Buela-Casal, 2010) han encontrado asociaciones entre las diferentes dimensiones de orientación temporal y determinados problemas de salud; por ejemplo, la orientación temporal presente y futura estarían relacionadas con la predicción del consumo de sustancias, la orientación al pasado se podría considerar como una variable moderadora en la percepción de experiencias traumáticas y la orientación futura sería una variable predictora de comportamientos asociados a estilos de vida saludables.

Con respecto a la relación entre la perspectiva temporal y la afectividad, Díaz, Toboso, Dávila, Escribano y Delgado (2008) refieren los resultados de estudios correlacionales entre las dimensiones temporales propuestas por Zimbardo y el bienestar subjetivo -el cual contempla las afectividades positiva y negativa. Estos autores reportan una correlación negativa y significativa entre los puntajes de la dimensión de pasado negativo y el bienestar subjetivo. Asimismo, las dimensiones del presente hedonista y del futuro presentaban una correlación positiva y significativa con bienestar subjetivo. Estos mismos autores, al realizar un análisis de regresión múltiple, destacan las siguientes cuatro dimensiones como predictoras del bienestar subjetivo: Pasado negativo, presente hedonista, futuro y presente fatalista. Por su parte, Oyanadel y Buela-Casal (2010) encuentran nuevamente al pasado negativo como una dimensión temporal relevante en el bienestar, al encontrar una correlación negativa y significativa con puntajes sobre percepción en el estado de salud física y mental.

En los últimos años, el enfoque de la psicología positiva ha puesto énfasis en el estudio de factores que incrementen los estados emocionales positivos. Este enfoque salutogénico ha sido señalado por algunos autores como una perspectiva más amplia para el estudio psicológico del ser humano y para la comprensión de determinadas enfermedades mentales como la depresión (Hervás, Sánchez \& Vázquez, 2008; Labiano, 2007). Este auge también ha permitido identificar algunos de estos elementos como factores protectores contra la depresión, entre los que se incluyen la afectividad y la orientación temporal. Identificar el impacto y relación que tienen estos elementos en grupos con alta presencia de depresión, como son los adolescentes, posibilitaría el desarrollo de propuestas para su prevención y atención.

El objetivo de la presente investigación es identificar el perfil y nivel de depresión, afectividad y orienta- 
ción temporal de los estudiantes de bachillerato de la muestra. Asimismo, se identifica que elementos de la afectividad pueden considerarse como predictores de la depresión y, a su vez, que dimensiones temporales predicen los índices de afectividad.

\section{MÉTODO}

\section{Participantes}

Se trabajó con una población de estudiantes de bachillerato del Colegio de Ciencias y Humanidades $(\mathrm{CCH})$ plantel Azcapotzalco, de la Ciudad de México. La muestra fue de tipo no probabilística y estuvo constituida por 50 estudiantes (35 mujeres y 15 hombres). Su edad oscilaba entre los 16 y 18 años. El diseño de la investigación es descriptivo, correlacional y ex post facto retrospectivo.

\section{Instrumentos}

Escala de afecto positivo-negativo (PANAS) (Robles \& Paez, 2003). Instrumento tipo likert conformado por 20 ítems que contienen adjetivos asociados a estados afectivos positivo y negativo. Evalúa tanto la afectividad-rasgo (las dos últimas semanas) como la afectividad-estado (cómo regularmente se siente el sujeto), al elegir entre un rango de variación que oscila del 1 (nada) al 5 (mucho). El instrumento proporciona un puntaje general de afectividad, obtenido a partir del puntaje de afectividad positiva, del cual se resta el puntaje de afectividad negativa. Los puntajes generales que tengan como resultado un número positivo tienden a mostrar una afectividad positiva en los sujetos, mientras que los resultados con números negativos son indicativos de afectividad negativa.

Inventario de depresión de Beck-II (BDI-II) (Beck, Steer \& Brown, 2006). Cuestionario tipo likert conformado por 21 ítems con cuatro opciones de respuesta que corresponden a diferentes niveles de depresión. El puntaje total identifica los siguientes niveles de depresión: mínimo (0-13 puntos); leve (14-19 puntos); moderado (20-28 puntos); grave (29-63 puntos). Además del puntaje general de depresión, este instrumento se divide en dos áreas: a) depresión somática y b) depresión cognitiva.

Inventario de perspectiva temporal de Zimbardo (ZTPI) (Zimbardo \& Boyd, 2009). Es una escala tipo likert de 56 ítems con cinco opciones de respuestas cada una. Evalúa cinco dimensiones temporales: a) pasado positivo, b) pasado negativo, c) presente fatalista, d) presente placentero, y e) futuro.

\section{Procedimiento}

Previa autorización del área de psicología del $\mathrm{CCH}$ Azcapotzalco, se solicitó a los estudiantes seleccionados su consentimiento para su participación en la investigación y la respuesta a los instrumentos. Se les informó acerca del manejo confidencial de los datos y que su fin era exclusivamente investigativo. La aplicación de los instrumentos se realizó de manera grupal en un aula del CCH. Después se capturaron los datos ${ }^{2}$ y se realizaron análisis descriptivos, correlacionales con el paquete estadístico IBM-SPSS-21, así como dos análisis de regresión múltiple; el primero de ellos tuvo como objetivo identificar qué elementos son predictores para la depresión. En el segundo análisis de regresión se identificaron qué factores temporales eran predictores de la variable afectividad-estado.

\section{RESULTADOS}

\section{Análisis descriptivos}

En la variable depresión la mayoría de los participantes presentan un nivel mínimo (60\% de la muestra), seguidos de aquellos que presentan un nivel leve (26\%), y en menos proporción se presentan niveles moderados (12\%) y severos de depresión (2\%). Por otra parte, el puntaje promedio de los participantes fue de 11.16, que correspondería a un nivel mínimo de depresión.

En afectividad se encontró un puntaje promedio con signo positivo tanto en la afectividad-estado como a la afectividad-rasgo (ver tabla 1). Estos puntajes positivos indican que existe un predominio de los estados afectivos positivos sobre los negativos tanto a nivel afectividad-rasgo como a nivel afectividad-estado, por lo que la muestra estudiada se caracterizaría por tener una tendencia a sentir energía, entusiasmo, estar alerta y gratificación al realizar actividades en su vida.

\footnotetext{
${ }^{2}$ Se agradece la participación de la Lic. Diana Nayeli Camacho Roldán para la captura de datos de los instrumentos aplicados en la presente investigación.
} 
Tabla 1. Promedio de afectividad-estado y afectividadrasgo

\begin{tabular}{c|c|c} 
& \multicolumn{2}{|c}{ Afectividad } \\
\hline & $\begin{array}{c}\text { Estado } \\
\text { (últimas dos semanas) }\end{array}$ & $\begin{array}{c}\text { Rasgo } \\
\text { (por lo general) }\end{array}$ \\
\hline Promedio & 10.92 & 13.86
\end{tabular}

En la variable de perspectiva temporal, los participantes muestran mayores puntajes promedios en las dimensiones temporales asociadas a aspectos positivos: a) Pasado positivo; b) Presente placentero; y c) Futuro. Mientras que se presentaron puntajes menores en las dimensiones asociadas a aspectos negativos: a) Pasado negativo; y b) Presente fatalista (ver tabla 2).

Esta combinación de puntajes presentado por los participantes es similar al perfil óptimo sugerido por Zimbardo y Boyd (2009): altos puntajes en pasado positivo, futuro y presente placentero y bajos en pasado negativo y presente fatalista, lo que indica que los sujetos de esta muestra tienden a tener un sentido de continuidad debido a la valoración de la familia, tradiciones, aspectos culturales (relacionado con su alto puntaje en el pasado positivo); una energía y alegría por el momento actual y la vida (por su alto puntaje en el presente placentero); y una visualización esperanzada, optimista y de agencia acerca del futuro (por su puntaje alto en el futuro).

\section{Análisis correlacionales}

La variable depresión en relación con la variable afectividad mostró correlaciones negativas y significativas con los dos tipos de afectividad (estado y rasgo), lo que significa que entre mayores puntajes en afectividad disminuyen los puntajes de depresión. Es relevante señalar que los coeficientes de correlación entre depresión y afectividad-estado fueron altos y moderados entre depresión y afectividad-rasgo, lo que llevó a presuponer que ambos tipos de afectividad podrían ser predictores de la depresión (ver tabla 4).

En las correlaciones de la variable de orientación temporal y afectividad sólo se encontraron correlaciones positivas y significativas entre la dimensión del pasado negativo tanto con la afectividad-estado $(\mathrm{r}=-.385 ; \mathrm{p}=.006)$ como con la afectividad-rasgo $(\mathrm{r}=$ $-.361 ; \mathrm{p}=.010)$. Esta misma dimensión de pasado negativo presentó una correlación positiva y significativa con la variable depresión $(\mathrm{r}=.417 ; \mathrm{p}=.003)$.

Análisis de regresión

A partir de las correlaciones encontradas se realizaron dos análisis de regresión múltiple con el método por pasos sucesivos (stepwise). En el primero de ellos la variable dependiente fue la depresión y las variables independientes fueron los dos tipos de afectividad (estado y rasgo) y las cinco dimensiones de orientación temporal. A partir del análisis estadístico se encontró que sólo la afectividad-estado tiene un valor predictivo relevante en la depresión. Los resultados muestran que la afectividad-estado explica el $48.9 \%$ de la variación

Tabla 2. Promedio de las dimensiones de orientación temporal

\begin{tabular}{c|c|c|c|c|c} 
& Pasado positivo & Presente placentero & Futuro & Pasado negativo & Presente fatalista \\
\hline Promedio & 3.67 & 3.46 & 3.42 & 3.04 & 2.84
\end{tabular}

Tabla 4. Correlaciones entre afectividad y depresión

\begin{tabular}{c|c|c|c|c}
\multirow{2}{*}{} & \multicolumn{2}{|c|}{ Estado (últimas dos semanas) } & \multicolumn{2}{|c}{ Rasgo (por lo general) } \\
\cline { 2 - 5 } & $\mathrm{R}$ & $\mathrm{P}$ & $\mathrm{R}$ & $\mathrm{P}$ \\
\hline Depresión & -.707 & .001 & -.594 & .001
\end{tabular}


del nivel de depresión, lo cual representa un porcentaje considerable de explicación (beta= -.456; $\mathrm{p}=.001$ )

A partir de los resultados encontrados en el primer análisis de regresión se evaluó si la variable afectividad-estado -la cual se identificó como predictora de la sintomatología depresiva- a su vez podría ser explicada por las dimensiones de orientación temporal. Los análisis estadísticos mostraron que las dimensiones de pasado negativo (beta $=-.625 ; \mathrm{p}=.002$ ) y de presente hedonista (beta $=.450 ; \mathrm{p}=.043$ ) explican el $18.7 \%$ de la variación del nivel de afectividad-estado. Aunque este porcentaje de explicación se puede considerar bajo, se debería contemplar el seguimiento de estas variables, sobre todo en el caso de la dimensión de presente negativo, que además mostró una correlación moderada con depresión.

\section{DISCUSIÓN}

Los resultados acerca de la depresión indican que la gran mayoría de los participantes sólo presentan algunos síntomas depresivos, por lo que se sitúan en un nivel mínimo o leve. Únicamente un pequeño porcentaje de la muestra estudiada podría considerarse que presenta todo el conjunto de síntomas necesario para considerársele como un trastorno depresivo. Aunque el porcentaje de sujetos con depresión severa es bajo -2\%- si lo sumamos al porcentaje de participantes que presentan una depresión moderada, el resultado es acorde con los porcentajes de prevalencia encontrados en otras poblaciones, los cuales oscilan entre el 5 y el 21\% (Berenzon et al., 2013). A pesar de la poca presencia de sujetos con niveles de depresión graves, es importante dar un seguimiento a sujetos que presentan niveles moderados o leves, ya que se ha señalado que en ocasiones por el solo hecho de presentar un cuadro incompleto de depresión y no ser diagnosticados con un trastorno depresivo se deja de prestar atención a las problemáticas asociadas con los síntomas, los cuales pueden llegar en un futuro a evolucionar en un nivel más intenso de depresión. Por otra parte, también es importante identificar lo más tempranamente posible los casos que se presentan niveles graves, para proporcionarles atención especializada de manera oportuna.

Los índices de afectividad señalan que la muestra se caracteriza por un estado afectivo positivo, lo cual concuerda con los porcentajes mínimos de depresión encontrados en los participantes del presente estudio. Asimismo, los resultados indican que la muestra se caracteriza por el perfil óptimo de tiempo propuesto por Zimbardo y Boyd (2009) - puntajes altos en pasado positivo, futuro y presente placentero y bajos en pasado negativo y presente fatalista-, lo que nuevamente estaría asociado al bajo porcentaje de población. Esto coincide con los resultados encontrados en otras investigaciones (Díaz et al., 2008), donde se encontró que la presencia de este perfil óptimo está asociado con una salud mental positiva.

En cuanto a las correlaciones encontradas, los resultados muestran correlaciones positivas entre afectividad -tanto estado como rasgo- con los puntajes de depresión. Es importante recordar que para el presente estudio se consideró el puntaje general de afectividad -el cual contempla tanto los puntajes de afectividad positiva como los de afectividad negativa. Esto significa que mientras más altos sean los puntajes de afectividad-que serían resultado de altos índices de afectividad positiva y bajos de negativa- menores serán los puntajes de depresión. Estos resultados coinciden con el modelo propuesto por Clark y Watson (citados en Torres et al., 2013) el cual señala que la depresión tiene como característica distintiva altos índices de afectividad positiva y bajos índices de afectividad negativa.

En cuanto a las dimensiones temporales que mostraron una correlación estadísticamente significativa con la afectividad y la depresión, se encontró sólo la del pasado negativo. Estos resultados indican que entre mayor tendencia presentan los sujetos a centrarse de manera negativa en el pasado, su afectividad tenderá a disminuir y volverse más negativa. Por otra parte, entre mayor tendencia a centrarse de manera negativa en el pasado, se elevan los puntajes de depresión. En investigaciones realizadas acerca de la orientación temporal, se han encontrado otras dimensiones -por ejemplo, el presente hedonista y el futuro- asociadas a elementos similares con la afectividad -como el bienestar subjetivo- o a ausencia de trastornos mentales -como la salud mental-. Una dimensión que presenta correlaciones significativas de manera consistente con otras variables asociadas a la afectividad es la de pasado negativo, lo cual es coincidente con los resultados del presente estudio (Díaz et al., 2008; Oyanadel \& 
Buela-Casal, 2010). De acuerdo con dichas investigaciones, esta visión negativa del pasado es un factor de riesgo para el desarrollo de trastornos mentales como la depresión, además de que dificulta la consecución de niveles óptimos de bienestar.

Finalmente, a partir de los análisis de regresión realizados se encontró que el índice de afectividad que presentan los sujetos en las dos semanas más recientes se puede considerar como un buen predictor de la presencia de depresión. De manera específica, el análisis de regresión encontrada mostró que a menor afectividad -presencia de mayores estados afectivos negativos y menores positivos- en las dos últimas semanas, la presencia de síntomas depresivos aumenta. Esto de nueva cuenta coincide con el ya mencionado modelo de Clark y Watson donde altos niveles de afecto negativo y bajos de positivo caracterizan la depresión. Asimismo, la combinación del pasado negativo y de presente hedonista sería un predictor de la afectividad. En concreto, con una menor tendencia a centrarse de manera negativa en el pasado y una mayor tendencia a disfrutar el presente, se presentarían mayores puntajes de afectividad; es decir, tenderían a presentarse mayores estados positivos de afectividad. Estos resultados coinciden con los realizados por Díaz et al., (2008), donde en estudios correlacionales también señalan al pasado negativo y al presente hedonista como dimen-

\section{REFERENCIAS}

Asociación Psiquiátrica Americana, (2014). Guía de consulta de los criterios diagnósticos del DSM-5. Madrid: Panamericana.

Beck, A., Steer, R. \& Brown, G. (2006). BDI-II. Inventario de depresión de Beck. Buenos Aires: Paidós.

Berenzon, S., Lara, M. A., Robles, R. \& Medina-Mora, M. E. (2013). Depresión: estado del conocimiento y la necesidad de políticas públicas y planes de acción en México. Salud Pública México, 55(1), 74-80.

Díaz, J. F., Toboso, M., Dávila, C., Escribano, C. \& Delgado, P. (2008). Bienestar, orientación temporal y estrategias de control en personas mayores: un estudio exploratorio. Revista de Psicología, UCA, 4(8), 79-95

Díaz-Morales, J. F. (2008). Estructura factorial y fiabilidad del Inventario de Perspectiva Temporal. Psicothema, 18(3), 565-571. siones asociadas a estados afectivos constituyentes al bienestar subjetivo.

Los resultados de nuestra investigación muestran la importancia de algunos factores de las variables de afectividad y orientación temporal en la presencia de sintomatología depresiva. Esto sugiere que podría prevenirse la aparición de sintomatología depresiva a través del desarrollo de estrategias que aumenten los estados afectivos positivos, así como la capacidad de disfrutar los eventos del presente y que disminuyan la tendencia a centrarse de manera negativa en situaciones del pasado. En este sentido, se sugiere trabajar con los estudiantes y planear actividades específicas para trabajar estos factores; por ejemplo, realizando talleres con actividades de revisión de vida donde se identifiquen aspectos valiosos en ella, modificando ideas erróneas acerca de vivencias del pasado, además de incrementar la visión de pasado positivo al construir redes sociales positivas y consistentes con los demás integrantes de la comunidad escolar y familiar; la dimensión del futuro se vería incrementada con actividades de establecimiento de metas o proyectos de vida y profesionales (Zimbardo \& Boyd, 2009). Para concluir, se sugiere seguir investigando en poblaciones similares la relación entre las variables estudiadas en el presente trabajo para identificar la consistencia de los resultados obtenidos. •

Fernandez, I., Ubillos, S. \& Zubieta, E. (2000). Manual de psicología social y salud, instrumentos de prácticas de investigación. San Sebastián: Universidad del País Vasco.

Hervás, G., Sánchez A. \& Vázquez, C. (2008). Intervenciones psicológicas para la promoción del bienestar. En: C. Vázquez \& G. Hervás (eds.). Psicología positiva aplicada (pp. 41-71). Bilbao: Desclée de Brouwer.

Labiano, M. (2007). Introducción a la psicología de la salud. En L. Oblitas. Psicología de la salud y calidad de vida (2a ed.) (pp. 2-21). México: Thomson.

Medina-Mora, M.E., Borges, G., Lara, C., Benjet, C., Blanco, J., Bautista, C. \& Aguilar-Gaxiola, S. (2003) Prevalencia de trastornos mentales y uso de servicios: Resultado de la encuesta nacional de epidemiología psiquiátrica en México. Salud Mental, (26)4, 1-16. 
Moral de la Rubia, J. (2011). La escala de afecto positivo y negativo (PANAS) en parejas casadas mexicanas. Ciencia ergo sum, 18(2), 117-125.

Oyanadel, C. \& Buela-Casal, G. (2010). La percepción del tiempo: influencias en la salud física y mental. Universitas Psychologica, 10(1), 149-161.

Paez, D. \& Carbonero, A. (1993). Afectividad, cognición y conducta social. Psicothema, (5), 133-150.

Rivadeneira, C., Dahab, J. \& Minici, A. (2013). El modelo cognitivo de la depresión. Revista de terapia cognitiva conductual, 22. Recuperado de http://cetecic.com.ar/ revista/el-modelo-cognitivo-de-la-depresion/

Robles, R. \& Paez, F. (2003). Estudio sobre la traducción al español y las propiedades psicométricas de las escalas de afecto positivo y negativo (PANAS). Salud Mental, (26)1, 69-75.

Sandín, B. (2003). Escala PANAS de afecto positivo y negativo para niños y adolescentes (PANASN). Revista de psicopatología y psicología clínica, 8(2), 173-182.
Schimelpfening, N. (2013). The DSM-5 and the Diagnosis of Depression. Recuperado de http://depression. about.com/od/diagnostictools/f/The-Dsm-5-AndThe-Diagnosis-Of-Depression.htm

Torres, L., Carbonell, M. M., Pérez, R. \& Riquelme, A. (2013). Afectividad positiva y negativa en ancianos con sintomatología depresiva, ansiosa o mixta. Revista del hospital psiquiátrico de La Habana, 10(2), 1-12.

Vázquez, C., Hervás, G. \& Hernangómez, L. (2008). Modelos cognitivos de la depresión: Su aplicación al estudio de las fases tempranas. En J. L. VázquezBarquero (Ed.), Las fases tempranas de las enfermedades mentales: Los trastornos depresivos, (pp. 21-33). Madrid: Elsevier.

Zimbardo, P. \& Boyd, J. (2009). La paradoja del tiempo. Barcelona: Paidós. 